\title{
COVID-19 and pregnant women: a letter to editor
}

\section{Letter to editor}

COVID-19 is a potentially dangerous respiratory disease that first detected in Wuhan, China in December 2019 and is spreading rapidly around the world. ${ }^{1}$ The small virus has caused great concern among various segments of the population, ${ }^{2}$ leading to more preventive actions such as frequent hand washing, disinfection of surfaces, use of masks and homemade quarantine.

One of the vulnerable groups that may be deeply concerned about COVID-19 is pregnant women. ${ }^{3}$ Searching through virtual groups and websites where pregnant women share information with their peers, we found that they had a lot of questions about the vague future that awaits them despite the coronavirus pandemic. Questions such as: How likely is it that pregnant women will develop QOVID-19? If a pregnant woman has the disease, what happened to her fetus? If a coronavirus test result is positive in a pregnant woman, is it necessary to terminate the pregnancy? What are the complications of pregnancy in pregnant women affected by COVID-19? In addition to such questions, which, due to lack of sufficient scientific evidence, do not yet have accurate answers, many pregnant women are also concerned that in such a situation, where home quarantine is highly recommended, go to the office of midwives, obstetricians or prenatal clinic of hospitals for routine visits, to get advice on common health problems in pregnancy, during childbirth and the puerperium and to check the results of laboratory tests and ultrasounds by their own physicians, has a potential risk of developing COVID-19.

Iran, like many other countries in the world, is facing the novel coronavirus disease. According to the official statistics of the Ministry of Health of Iran so far (April 10, 2020), 68192 positive coronavirus tests have been identified, and 4232 deaths have occurred due to COVID-19. ${ }^{4}$ During the outbreak of COVID-19 in Iran, in order to reduce the number of unnecessary visits of pregnant women to service providers, midwifery departments in some medical universities and midwives working in the private sector during a humanitarian action, have established online counseling groups on social media such as WhatsApp and Telegram and joined pregnant women who received prenatal care in health centers. The admins of these groups are experienced midwives with academic education, some of whom have master's and $\mathrm{PhD}$ degrees in midwifery and reproductive health. Their team provides free online counseling to pregnant women 24 hours a day, seven days a week on pregnancy, childbirth and puerperium issues. In emergencies, pregnant women are advised to go to a "clean hospital," which, according to the Ministry of Health's classification, does not admit patients suspected of having COVID-19. In addition, some faculty members in midwifery departments of universities answer questions of pregnant women as free telephone counseling groups called "Voice of Midwife." The goal is to reduce the unnecessary visits of pregnant women to medical centers, to cut off the coronavirus transmission chain and to reduce the preventable maternal mortality.
Volume 7 Issue 3 - 2020

\author{
Somayeh Makvandi,' Mitra Mahdavian,' Leila \\ Karimi $^{2}$ \\ 'Department of Midwifery, School of Nursing and Midwifery, \\ Islamic Azad University, Iran \\ ${ }^{2}$ Behavioral Sciences Research Center, Life style institute, \\ Nursing Faculty, Baqiyatallah University of Medical Sciences, Iran
}

Correspondence: Leila Karimi, PhD of Reproductive Health, Assistant Professor, Behavioral Sciences Research Center, Life style institute, Nursing Faculty, Baqiyatallah University of Medical Sciences, Iran, Email leilakarimi I47@gmail.com

Received: July 10, 2020 | Published: July 23, 2020

Many pregnant women are worried about the risk of developing COVID-19 on days when they will be hospitalized for childbirth, and some of them want to have a home birth. The fear is such that some pregnant women are reluctant to go to the hospital despite having preterm labor symptoms. In such an extremely special situation, it is recommended that arrangements be made for home birth after weighing the pros and cons.

\section{Funding}

None.

\section{Acknowledgments}

None.

\section{Conflicts of interest}

The authors declare that there are no conflicts of interest.

\section{References}

1. Huang C, Wang Y, Li X, et al. Clinical features of patients infected with 2019 novel coronavirus in Wuhan, China. The Lancet. 2020;395(10223):497-506.

2. Wang C, Pan R, Wan X, et al. Immediate psychological responses and associated factors during the initial stage of the 2019 coronavirus disease (COVID-19) epidemic among the general population in china. International Journal of Environmental Research and Public Health. 2020;17(5):1729.

3. Liang H, Acharya G. Novel corona virus disease (COVID-19) in pregnancy: What clinical recommendations to follow. Acta Obstet Gynecol Scand. 2020;99(4):439-442.

4. Official: 35,465 COVID19-infected patients recover in Iran. Iranian Ministry of Health and Medical Education; 2020. 